\title{
The Space Art of Scriven Bolton
}

\section{Clive Davenhall}

Abstract. Scriven Bolton (1883-1929) was an amateur astronomer from Leeds active in the first three decades of the twentieth century. He was also a skilled artist and commercial illustrator, specialising in astronomical subjects. He produced a substantial body of work which was reproduced in a wide range of publications, including newspapers, magazines and books published on both sides of the Atlantic. His work was intended to be scientifically accurate, and illustrates the consensus astronomical knowledge in the first decades of the twentieth century. No comprehensive list of his work was ever produced and he is now little remembered. This paper is a first report in an attempt to recover some his work and ultimately assess his contribution to early astronomical illustration.

\section{Introduction}

Thomas Simeon Scriven Bolton (1883-1929), always known as Scriven, was an amateur astronomer from Leeds active from the early years of the twentieth century until his death in 1929 (Figure 1). He was also a talented and prolific artist and his astronomical drawings and illustrations became well known. He died at the tragically early age of 46 and is little remembered now.

Published information about Bolton's life and work is scarce and fragmentary. The Royal Astronomical Society (RAS) published an obituary anonymously (as was often the case at the time), and there are recent papers by Armitage and Davenhall. ${ }^{1}$ Scriven Bolton's work appeared in a wide range of publications and no comprehensive list of it has ever been assembled. This paper is the first report in what is likely to be a protracted undertaking to assemble a collection of examples of his work and ultimately reassess his contribution to the development of astronomical illustration, or what is now often called 'space art'.

\footnotetext{
${ }^{1}$ Anonymous, 'Scriven Bolton', Monthly Notices of the Royal Astronomical Society, 90 (1930): p. 359 (obituary); J. Armitage, 'Scriven Bolton and his Giant Two-Foot Telescope', Society for the History of Astronomy Bulletin, No. 18 (2009): pp. 27-29; Clive Davenhall, 'The Space Art of Scriven Bolton', Society for the History of Astronomy Bulletin, No. 18 (2009): pp. 30-32.
}

Clive Davenhall, 'The Space Art of Scriven Bolton', eds. Nicholas Campion and Rolf Sinclair, Culture and Cosmos, Vol. 16 nos. 1 and 2, 2012, pp. 385-392. www.CultureAndCosmos.org 
386 The Space Art of Scriven Bolton

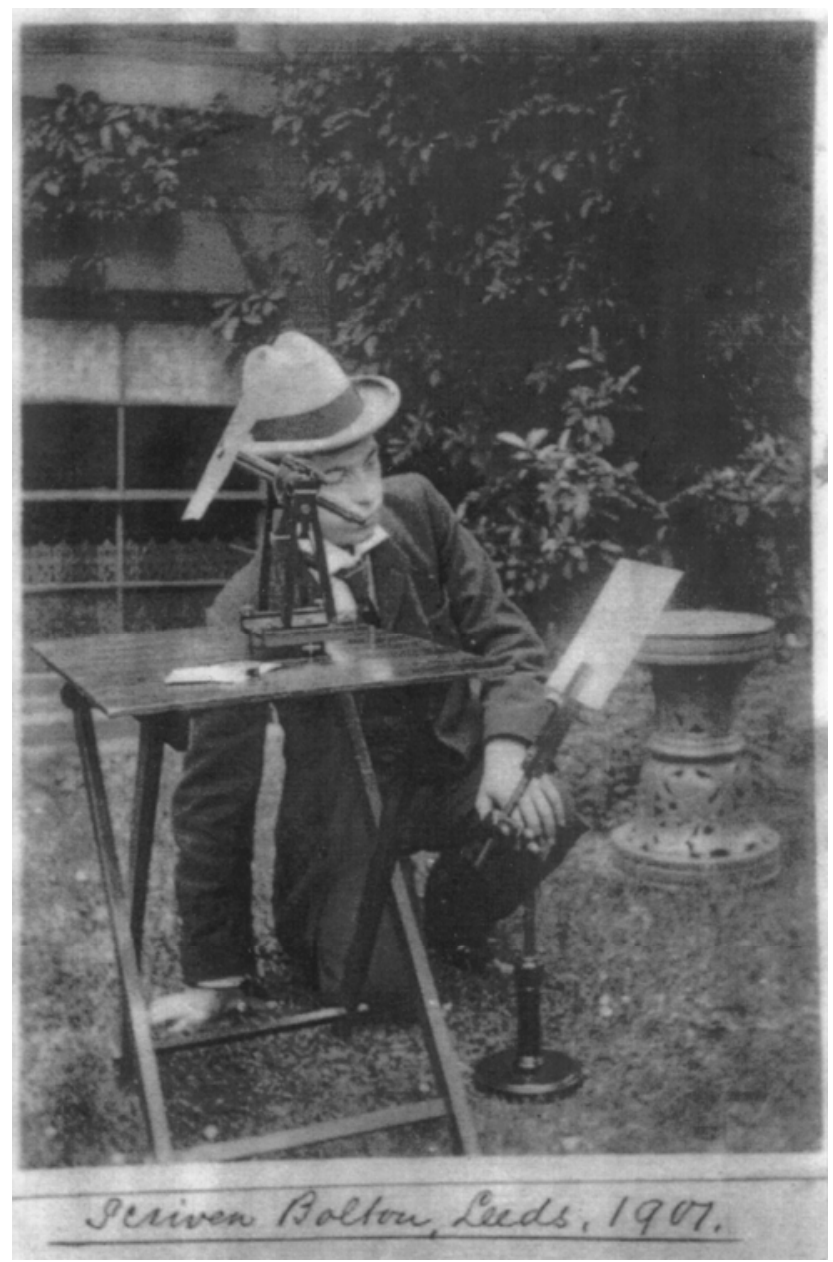

Figure 1. Scriven Bolton (1883-1929) in 1907, projecting a solar image with what appears to be a small portable transit instrument (from the archives of the Leeds Astronomical Society and reproduced courtesy of Raymond Emery).

\section{Life and Work}

Scriven Bolton's family were originally textile manufacturers but, in the late nineteenth century, his father bought into a mineral oil merchant business and Scriven duly followed him into this firm, which was his principal source of income. Astronomy, however, was his main interest and he set up an observatory with a ten-inch reflector adjacent to the 
family home. Around 1911 the family moved to Bramley in Leeds, and Scriven established another observatory, this time with a twenty-six-inch reflector. $^{2}$ Between 1906 and 1914 Leeds University's Duncombe Observatory was operated by volunteers from the Leeds Astronomical Society. ${ }^{3}$ Bolton made good use of this opportunity to use the eighteeninch reflector at the University Observatory.

In addition to the Leeds Astronomical Society he was a member of several other astronomical societies, including the RAS (joining in 1905) and the British Astronomical Association. He regularly published his observations in a variety of journals, including Nature, the Monthly Notices of the Royal Astronomical Society and the Journal of the British Astronomical Association. However, Bolton was best known for his astronomical drawings and illustrations (Figures 2 - 5). For fifteen years he was a member of staff of the Illustrated London News (ILN), regularly contributing astronomical drawings and sketches. He also had material published in The Graphic, The Sphere, Le Journal Astronomique de France and several papers in the United States. ${ }^{4}$ Some of his drawings were reproduced as book illustrations.

In addition to drawing he also painted in pastel (Figure 2). He was an accomplished artist, won numerous awards for his work and was a Fellow of the Royal Society of Arts. He had work exhibited in the Exposición General de Estudios Lunares held in Barcelona in 1912 and was awarded a certificate by the Astronomical Society of Barcelona for his contribution. ${ }^{5}$

\footnotetext{
${ }^{2}$ Armitage, 'Scriven Bolton and his Giant Two-Foot Telescope'.

${ }^{3}$ R. Hutchins, British University Observatories 1772-1939 (Aldershot: Ashgate, 2008), p. 419 and Table 7.4, pp. 416-17.

${ }^{4}$ It would be almost impossible to assemble a complete list now. However, as just one example, on 11 September 1927 the Sunday magazine section of the Milwaukee Sentinel-Telegram published on p. 5 a full-page article by Isabel M. Lewis about studying the Moon using polarised light that was illustrated with one of Scriven Bolton's drawings.

${ }^{5}$ Sociedad Astronómica de Barcelona, 'Exposición General de Estudios Lunares: Catálogo' (1912). See also C. Wood, Lunar Photo of the Day for 15 November 2006, available at URL: http://www.lpod.org/?m=20061115 [accessed 6 March 2011].
} 


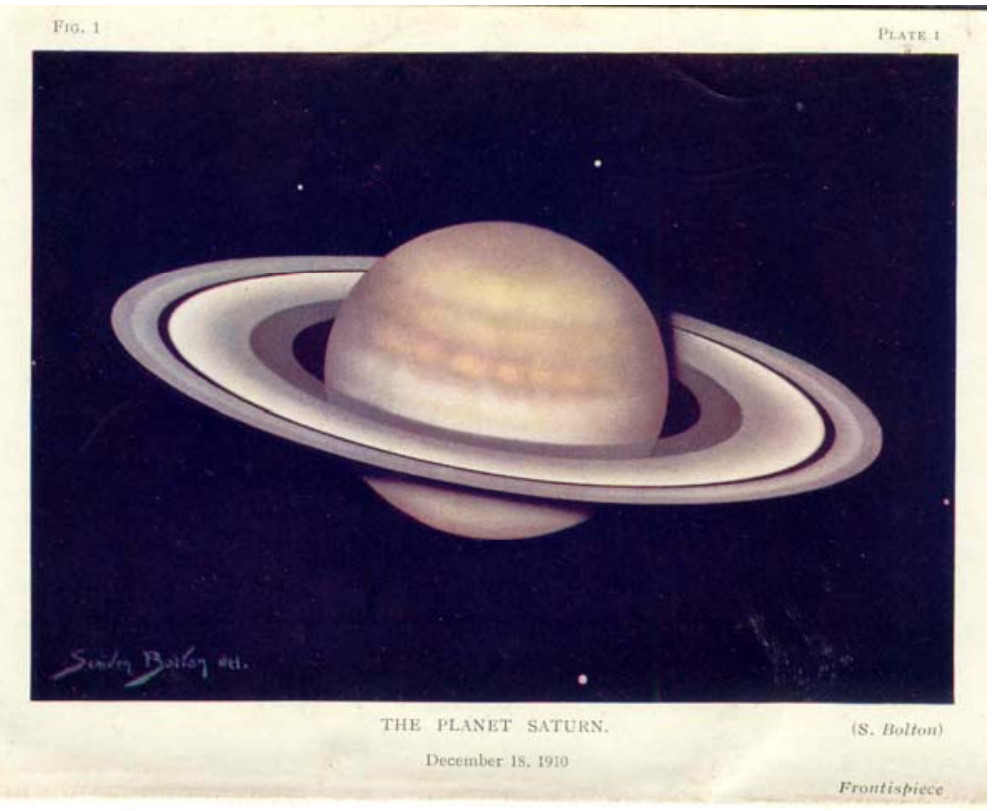

Figure 2. Saturn on 18 December 1910, from the frontispiece to G. F. Chamber's Astronomy (London: Hutchinson and Co, 1912); the copy reproduced here is courtesy of Raymond Emery

He published several letters in Nature, including two describing the aurora as seen from his home in Leeds on the nights of 5 November 1915 and 25 December 1918. Both letters were accompanied by drawings (Figure 3). ${ }^{6}$

Bolton developed an effective method for producing realistic lunar landscapes that involved making a model of the surface in plasticine or similar material, photographing it and then painting over the photograph. This approach was a development of the technique of modelling the lunar surface and photographing it under oblique light, which dates from the work of Nasmyth and Carpenter in the midnineteenth century (Figure 4). ${ }^{7}$

\footnotetext{
${ }^{6}$ S. Bolton, 'The Aurora Borealis of November 5', Nature, 96, No. 2403 (1915): p. 314 and 'The Aurora Borealis of December 25, 1918', Nature, 102, No. 2569 (1919): p. 405.

${ }^{7}$ T. A. Dobbins, 'Nasmyth, James Hall' (2007) in Biographical Encyclopaedia of Astronomers, ed. T. Hockey (New York: Springer, 2007), pp. 821-22.
} 
Clive Davenhall 389

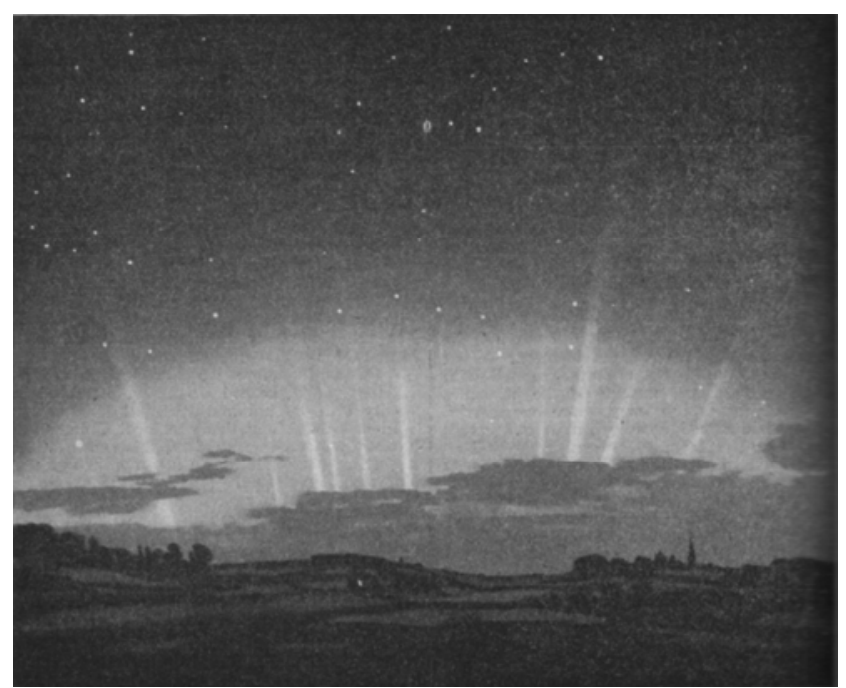

Figure 3. The aurora borealis, as seen from Bramley, Leeds on the night of 5 November $1915^{8}$

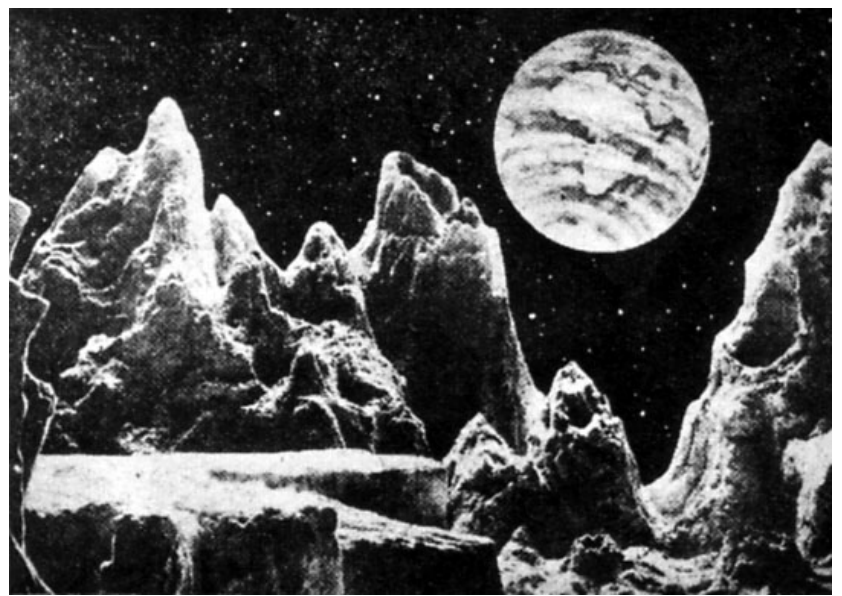

Figure 4. A lunar landscape with the earth in the background. This painting was from a photography of a model.

Between 1918 and 1924 Bolton published a series of thirteen articles in the American magazine Popular Science. ${ }^{9}$ These publications reveal a

${ }^{8}$ This illustration accompanied a letter published in Nature, 96, No. 2403 (1915): p. 314; reproduced courtesy of the Library of the University of Edinburgh.

Culture and Cosmos 
new side to his activities. Each article is illustrated with at least one of his drawings, but he also provided the accompanying text. In some cases the articles are short pieces comprising a single illustration and text which is really an extended caption. However, others are full-blown articles with substantial text and several illustrations. Popular Science was aimed at a general audience, and the articles treated then-contemporary topics in astronomy that would be of popular interest, such as speculations on whether the earth had a second moon, Svante Arrhenius' panspermia hypothesis and speculation on what might be hidden behind Venus' veil of clouds.

In 1928 T.E.R. Phillips and W. H. Steavenson published their compendious Splendour of the Heavens, with chapters contributed by various authors. ${ }^{10}$ This book was a comprehensive compilation of popular astronomy which proved extremely popular and influential. It was a lavish volume with nearly 1000 illustrations, including a few by Bolton. These drawings may have been some of his last work. Less than a year after their appearance he died on Christmas day 1929 of complications following influenza, at the tragically early age of 46 .

\section{Aftermath and Influence}

Bolton's mother wished to see a proper memorial to her son's work. To this end she wished to donate to Leeds University a fully functional observatory, including his twenty-six-inch telescope. However, the mirror for this instrument was missing (for reasons that remain unclear), so she commissioned a replacement from Cooke, Troughton and Simms. The new mirror, actually of twenty-three-inch diameter, was duly delivered, but never used; it is now in the care of the South Staffordshire Observatories Group. ${ }^{11}$ However, Leeds University still remembers Scriven Bolton in its annual Bolton lecture in which a distinguished speaker addresses some topic in contemporary astronomy before a public audience.

${ }^{9}$ An on-line archive of past issues of Popular Science is available on the magazine's website and includes the period when Scriven Bolton contributed articles. See: http://www.popsci.com/archives [accessed 6 March 2011].

${ }^{10}$ T.E.R. Phillips and W. H. Steavenson, eds., Hutchinson's Splendour of the Heavens (London: Hutchinson, 1928).

${ }^{11}$ Armitage, 'Scriven Bolton and his Giant Two-Foot Telescope'. 


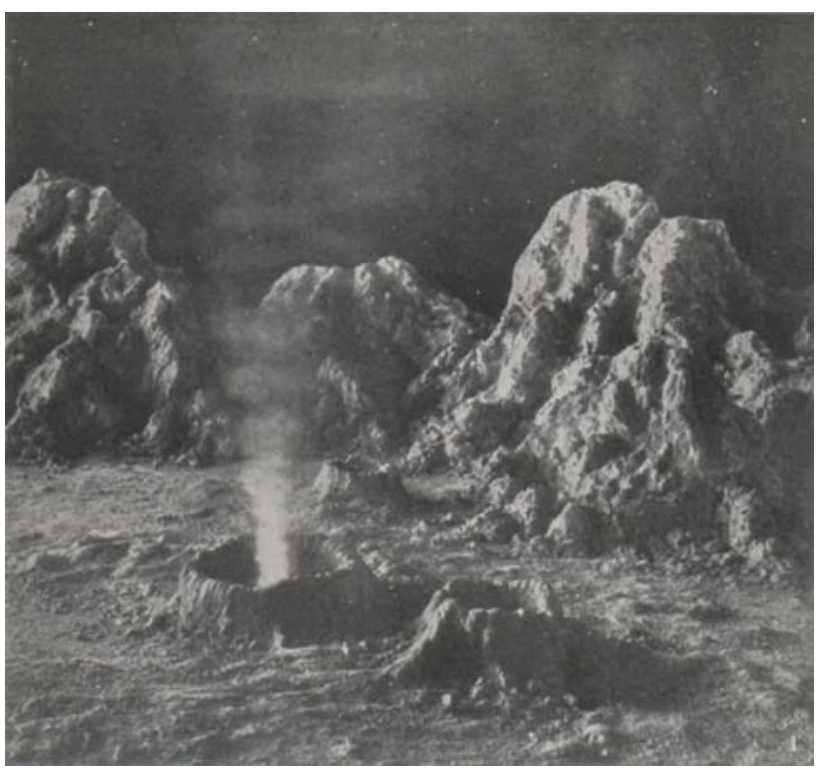

Figure 5. A lunar landscape showing a still-active volcano close to the wall of the crater Plato, painted from a model where the artist was credited as 'Bolton Scriven'. 12

Much space art naturally divides into three categories: depictions of astronomical objects and phenomena as seen through either the telescope or with the unaided eye; imagined landscapes on other worlds; technical illustrations, in which text and graphics, often superimposed on an underlying image, are used to convey astronomical ideas and information. Bolton produced work in all of these categories (Figures 2 5). Lunar landscapes were a favourite, but he also drew scenes on Mercury, Venus and Mars. In common with other serious astronomical illustrators, Bolton aimed to make his drawings and paintings scientifically accurate, and they illustrate the astronomical ideas of their day. The Moon, for example, is shown with volcanic craters and steepsided mountains (Figure 4), but these representations were virtually ubiquitous at the time.

12 The version reproduced here appeared in Harmsworth's Universal Encyclopaedia, ed. Sir John Hammerton (London: The Educational Book Co. Ltd, 1920-22), p. 5518 (Reproduced from a copy in the present author's collection.) 
Bolton's work was known to other early practitioners of astronomical illustration, or 'space art', such as Chesley Bonestell and Lucien Rudaux. Bolton and Bonestell met while both worked for the ILN (Bonestell lived in London during the 1920s). Indeed, it was Bolton who first introduced Bonestell to space art. Bonestell would later further refine the technique of making landscape models, photographing them and painting from the result. He seems to have been spurred initially, in part, by what he saw as deficiencies in Bolton's technique. ${ }^{13}$

Scriven Bolton's work appeared in a wide variety of publications and many of his drawings were reused in more than one publication. However, no comprehensive list has ever been compiled and much is now dispersed and lost. I hope that it will be possible to assemble a representative sample and consequently reassess his contribution to the early development of space art.

\section{Acknowledgements}

I am grateful to Ray Emery of Leeds Astronomical Society for first introducing me to Scriven Bolton's work, for providing me with copies of some of his illustrations and for useful discussions. I am also grateful to John Armitage of the South Staffordshire Observatories Group for useful discussions. I am pleased to acknowledge use of the Library of the Royal Observatory Edinburgh and the National Library of Scotland. Any mistakes remain my own.

${ }^{13}$ R. Miller and F. C. Durant III, The Art of Chesley Bonestell (London: Paper Tiger, 2001), p. 24; R. Miller, 'Chesley Bonestell: the Fine Art of Space Travel' in M. H. Schuetz, A Chesley Bonestell Space Art Chronology (Parkland, Florida: Universal Publishers, 1999), p. xvi, http://www.bookpump.com/upb/pdfb/1128290b.pdf [accessed 6 March 2011].

Culture and Cosmos 